Research Paper

\title{
Rho GTPase Rho] is Associated with Gastric Cancer Progression and Metastasis
}

\author{
Chan Kim ${ }^{1,2}$, Hannah Yang, ${ }^{3,4}$ Intae Park ${ }^{3,4}$, Hong Jae Chon ${ }^{1,2}$, Joo Hoon Kim5, Woo Sun Kwon ${ }^{6}$, Won Suk \\ Lee $^{6}$, Tae Soo Kim 6 , Sun Young Rha ${ }^{5,6,7 \bowtie}$ \\ 1. Medical Oncology, CHA Bundang Medical Center, CHA University, Seongnam, Korea; \\ 2. Laboratory of Tumor Vasculature and Microenvironment, CHA University, Seongnam, Korea; \\ 3. Asan Institute for Life Sciences, Seoul, Korea; \\ 4. Graduate School of Medical Science and Engineering, Korea Advanced Institute of Science and Technology, Daejeon, Korea; \\ 5. Division of Medical Oncology, Yonsei University College of Medicine, Seoul, Korea; \\ 6. Song-Dang Institute for Cancer Research; \\ 7. Brain Korea 21 Project for Medical Science, Yonsei University College of Medicine, Seoul, Korea.
}

$\triangle$ Corresponding author: Sun Young Rha, MD, PhD, Professor, Department of Internal Medicine, Yonsei University College of Medicine, 50 Yonsei-ro, Seodaemun-Ku, Seoul, Korea, 120-752. Phone: +82-2-2228-8050, Fax: +82-2-362-5592, E-mail: rha7655@yuhs.ac.

(C) Ivyspring International Publisher. Reproduction is permitted for personal, noncommercial use, provided that the article is in whole, unmodified, and properly cited. See http://ivyspring.com/terms for terms and conditions.

Received: 2016.03.18; Accepted: 2016.05.30; Published: 2016.07.08

\begin{abstract}
Rho GTPases play a pivotal role in tumor progression by regulating tumor cell migration and invasion. However, the role of Rho GTPases in gastric cancer (GC) remains unexplored. This study aimed to investigate the clinical implications of RhoJ, which is an uncharted member of Rho family. RhoJ expression in human GC cell lines and surgical specimens from GC patients were analyzed. Moreover, in vitro gain-of-function analysis was performed to evaluate the malignant phenotypes of RhoJ-overexpressing GC cells. The extent of Rhol expression varied among GC cell lines and GC patients. YCC-9 cell line displayed the strongest expression, while YCC-10, -11 , and -16 showed scant expressions. Of the $70 \mathrm{GC}$ patients, 34 (48.6\%) had Rhol expression in their GC tissue, and patients with high Rhol expression had more diffuse type GC (73.5\% vs. $41.7 \%)$, were at more advanced stages (stage III, IV: $85.3 \%$ vs. $58.4 \%$ ), and had more frequent metastasis (47.1\% vs. $11.1 \%$ ), denoting that RhoJ has a potential role in GC progression and metastasis. High RhoJ expression significantly correlated with poor overall survival and recurrence-free survival after surgical resection of gastric cancer. Finally, In vitro gain-of-function experiments showed $41.3 \%$ enhanced motility and $60.4 \%$ enhanced invasiveness in RhoJ-overexpressing GC cells compared to control, with negligible difference in cell proliferation. Collectively, high RhoJ expression is an independent negative prognostic factor for the survival outcome of GC and correlated with the increased cell motility and invasiveness.
\end{abstract}

Key words: RhoJ, Rho GTPase, gastric cancer, progression, metastasis.

\section{Introduction}

Gastric cancer (GC) is one of the most prevalent malignancies worldwide [1]. Despite recent therapeutic advances, the overall prognosis of recurrent or metastatic GC remains dismal, thereby necessitating the unearthing of novel therapeutic targets in GC [2-4].

The invasive and migratory capability of malignant tumor cells into neighboring microenvironment is the most critical step that governs the entire process of tumor progression $[5,6]$.
Among the prime mediators which are responsible for tumor cell migration and invasion, one particular family of proteins that seems to play a decisive role is Rho GTPase [7-9]. They are molecular switches which regulate various intracellular processes such as cytoskeletal rearrangement, thereby playing important roles in every aspects of tumor progression including malignant transformation, proliferation, invasion, and metastasis of tumor cells [8-10]. Among more than 20 members of Rho family, RhoA and Rac1 
are most extensively studied where their overexpression was found to be closely related to GC progression, while other members are largely uncharted [10].

RhoJ is a recently identified member of Rho family [11, 12]. Although RhoJ shares structural similarities and common effector molecules with other Rho GTPases, it has other distinct characteristics [12-14]. Recently published study demonstrates that RhoJ is enriched in endothelial cells and plays a positive angiogenic role during tumor progression and wound healing [15]. However, little is known regarding the role of RhoJ in non-endothelial cells such as tumor cells. Intriguingly, few recent studies have been published regarding the expression of RhoJ in melanoma cells and addressed its role in the regulation of melanoma cell migration and invasion $[16,17]$. Considering these findings, we set out to investigate the possible role of RhoJ during GC progression.

In the present study, we aimed to demonstrate the correlation between RhoJ expression and clinicopathologic parameters as well as to uncover the putative role of RhoJ in regulating GC cell behavior.

\section{Materials and Methods}

\section{Cell culture and transfection}

Human gastric cancer cell lines, YCC-1, YCC-2, YCC-3, YCC-7, YCC-9, YCC-10,YCC-11, and YCC-16 were established by Yonsei Cancer Center (Seoul, Korea) from GC patients through the isolation of blood (YCC-16) or ascites (others cell lines). Cells were incubated in RPMI-1640 (Welgene) with 10\% fetal bovine serum (FBS) in $5 \% \mathrm{CO}_{2}$ at $37^{\circ} \mathrm{C}$. Human umbilical vein endothelial cells (HUVEC, Lonza) were cultured in endothelial growth medium (EGM-2, Lonza). Full-length human RhoJ expression vector and an empty vector were acquired from Addgene (http://addgene.org). YCC-16 cells were transfected with either the RhoJ expression vector or empty vector by using Lipofectamine LTX (Life Technologies) following the manufacturer's instruction.

\section{Western blotting}

Cell lysates were separated with SDS-PAGE and transferred to PVDF membranes (Millipore). The membranes were blocked with 5\% skim milk, followed by the incubation with the following primary antibodies: RhoJ (mouse monoclonal, Abnova) and GAPDH (rabbit polyclonal, Santacruz). The bound antibodies were visualized with HRP-conjugated secondary antibodies. Signals were developed with HRP substrate and detected with LAS-1000 (Fuji film).

\section{Proliferation assay}

The cells were placed in a 96-well plate at a concentration of $5 \times 10^{3}$ cells per well. After incubation for 1 to 3 days, the amount of viable cells was determined using the water-soluble tetrazolium assay (WST) kit (DaeilLab). After incubating the cells with WST solution for $20 \mathrm{~min}$ at $37^{\circ} \mathrm{C}$, optical absorbance was measured with a microplate reader (Bio-Rad).

\section{Migration assay}

$4 \times 10^{4}$ cells were seeded in serum-free medium onto the upper chamber of 24-well culture inserts with $8 \mu \mathrm{m}$ pores (Millipore) and medium containing $5 \%$ FBS was placed in the lower chamber. After $24 \mathrm{hr}$, the cells on the upper surface of membrane were removed with a cotton swab. The cells that had migrated to the lower surface of membrane were fixed and visualized with $1 \%$ crystal violet (Sigma).

\section{Invasion assay}

Cell invasion was compared using BioCoat Matrigel $^{\mathrm{TM}}$ invasion chambers (BD). $4 \times 10^{4}$ cells were plated in the upper chamber in serum-free medium and the lower chamber was filled with medium containing 10\% FBS. After 36 hours, the cells on the upper surface were removed with a cotton swab. The cells that had migrated through the Matrigel ${ }^{\mathrm{TM}}$ invasion chamber were fixed and visualized with $1 \%$ crystal violet.

\section{Patients and surgical specimens}

70 patients who were diagnosed with primary gastric adenocarcinoma and received radical gastrectomy were enrolled at Yonsei Cancer Center, Severance Hospital (Yonsei University Health System, Seoul, Korea). The clinicopathologic variables including age, sex, histologic type, Lauren's classification, stage, type of surgery, and survival outcome were reviewed retrospectively based on the electronic medical records. Pathological diagnosis and staging of GC was made according to the American Joint Committee on Cancer 2010 staging system. The study was approved by the institutional review board.

\section{Immunohistochemical and immunofluorescent staining}

Immunohistochemical (IHC) staining was performed on the surgical specimens of GC patients. Slides were antigen-retrieved by autoclaving and blocked. Samples were incubated with RhoJ antibody (mouse monoclonal clone 1E4, Abnova) overnight, followed by incubation with biotinyated secondary antibody. Slides were reacted with Vectastatin ABC reagent (Vector laboratories) and DAB (DAKO) was 
used as a chromogen. The following scoring system was used to grade RhoJ immunoreactivity: score $0=$ no staining or non-specific staining of tumor cells; score $1=$ weak and incomplete staining of $>10 \%$ of tumor cells; score $2=$ moderate and complete staining of $>33 \%$ of tumor cells; score $3=$ strong and complete staining of $>67 \%$ of tumor cells. Those with $>10 \%$ positive stained tumor cells (scores 1 to 3 ) were regarded to be RhoJ-positive. RhoJ immunoreactivity in endothelial cells was excluded from analysis. For immunofluorescent (IF) staining, cells cultured on coverslips were blocked and incubated overnight with the phospho-histone H3 antibody (Cell Signaling). Then, slides were incubated with cy3-conjugated secondary antibodies (Jackson ImmunoResearch). Nuclei were stained with DAPI (Invitrogen) and F-actin was stained with Acti-stain Phalloidin (Cytoskeleton). Then the slides were mounted and imaged with a LSM510 confocal microscope (Carl Zeiss).

\section{Statistical analysis}

Survival curves were estimated with the Kaplan-Meier method, and the statistical differences were determined using the log-rank test. Overall survival (OS) was calculated from the date of gastrectomy to the date of death, while recurrence-free survival (RFS) was defined as the interval between the date of gastrectomy and the date of either recurrence or death. Multivariate analyses were performed with the Cox proportional hazard model. Chi-square tests and independent sample t-test were used for the analysis of variables. All statistical analyses were carried out with SPSS version 12.0 (SPSS, Inc.).

\section{Results}

\section{Human GC cell lines express RhoJ differently}

At first, we compared the expression of RhoJ proteins in various human GC cell lines and HUVECs using immunoblotting. The expression levels of RhoJ differed among different GC cell lines, with the highest in YCC-9 cell line, in which the expression level of RhoJ was higher than that of human endothelial cell, HUVEC, while being barely detectable in YCC-10, -11, and -16 cell lines (Figure 1A). These findings suggest that human GC cells also express RhoJ as well as endothelial cells.

\section{High RhoJ expression is associated with diffuse type GC at later stages and poor survival outcome as well as more frequent distant metastasis}

To evaluate the expression of RhoJ in GC tissues in addition to the cell lines, we performed IHC staining for 70 cases of surgically resected GC specimens. IHC staining results displayed RhoJ expression in GC tissues with varied intensities (Figure 1B). When the intensity of RhoJ expression was graded as negative ( $\leq 10 \%$ of cells stained) or positive (>10\% of cells stained), of the 70 GC tumor specimens, $34(48.6 \%)$ displayed the expression of RhoJ, while $36(51.4 \%)$ did not show any expression of RhoJ. We were able to observe the strong expression of RhoJ in cytoplasm of tumor cells, while it was occasionally expressed in tumor vasculatures (Figure 1C).

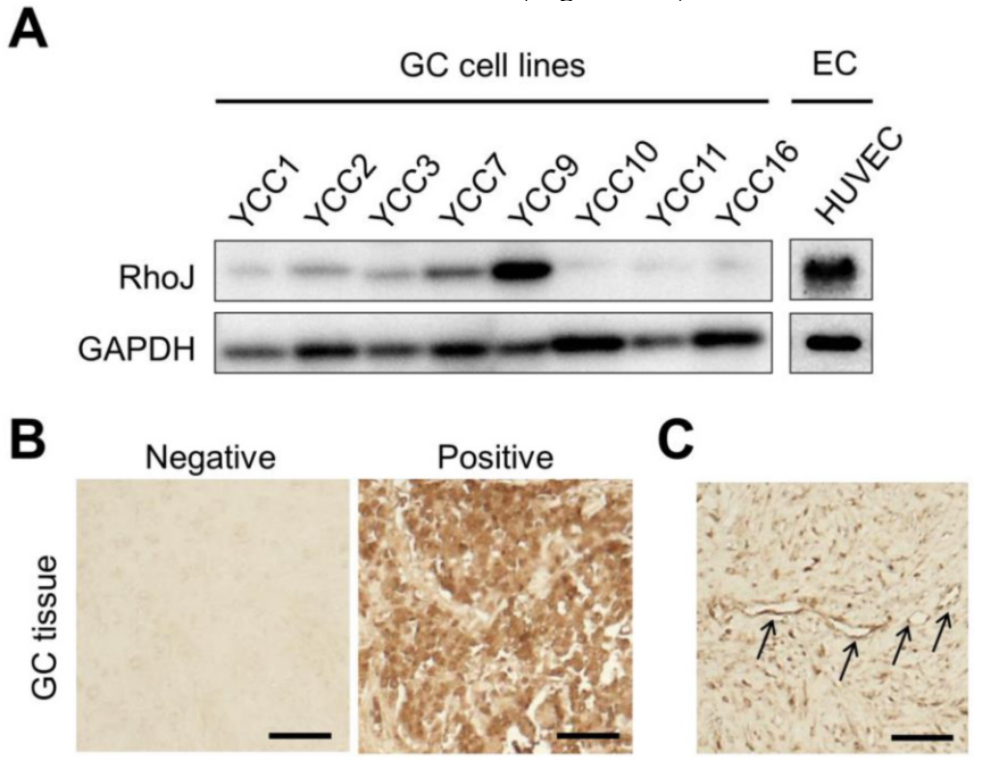

Figure 1. RhoJ expression in GC cell lines and surgical specimens of GC patients. (A) Various GC cell lines and HUVECs were cultured for 24 hr and harvested. Immunoblotting showing differential levels of RhoJ expression in various GC cell lines and HUVEC. EC, endothelial cell. (B) Immunohistochemical staining showing RhoJ expression in GC tissue. Scale bar: $200 \mu \mathrm{m}$. (C) Endothelial expression of RhoJ in GC tissue. Arrows indicate the endothelial expression of RhoJ. Scale bar: $200 \mu \mathrm{m}$. 
The baseline characteristics of the patient cohort are summarized in Table 1 . The median age at the time of GC diagnosis was 62 years (range 33-83) and the male-to-female ratio was 1.9. The most common histologic type was tubular adenocarcinoma (70\%) and $57.1 \%$ of tumors were classified as diffuse type by Lauren's classification. Almost all patients (94.3\%) received adjuvant chemotherapy, while only 2 out of 70 patients $(2.9 \%)$ underwent adjuvant radiotherapy. The median follow-up duration was 47.5 months and $47.1 \%$ of patients were alive at the time of analysis.

To characterize the features of GC patients with RhoJ expression, various clinicopathologic parameters were compared according to the RhoJ expressing status (Table 2). Patients with RhoJ-positive tumors had more diffuse type GC $(73.5 \%$ vs. $41.7 \%$, $p=0.007)$ at later stages (stage III, IV: $85.3 \%$ vs. $58.4 \%, p=0.023$ ) and distant metastasis ( $47.1 \%$ vs. $11.1 \%, p=0.001)$, denoting that RhoJ has a positive role in tumor progression and metastasis. In addition, RhoJ-positive tumors tended to be more poorly differentiated adenocarcinoma and signet ring cell carcinoma compared to RhoJ-negative tumors ( $82.4 \%$ vs. $52.8 \%$, $\mathrm{p}=0.043)$. However, there were no significant differences in age, sex, T stage, and N stage between RhoJ-positive and RhoJ-negative GC patients. Moreover, the proportion of patients receiving adjuvant chemotherapy or radiotherapy was not different between RhoJ-negative and RhoJ-positive groups.

Table 1. Baseline characteristics of the cohort.

\begin{tabular}{|c|c|}
\hline & $\mathrm{N}(\%)(\mathrm{n}=70)$ \\
\hline Age (median, range) & $62(33-83)$ \\
\hline \multicolumn{2}{|l|}{ Sex } \\
\hline Male & $46(65.7)$ \\
\hline Female & $24(34.3)$ \\
\hline \multicolumn{2}{|l|}{ Histology } \\
\hline WD & $3(4.3)$ \\
\hline MD & $17(24.3)$ \\
\hline PD & $29(41.4)$ \\
\hline SRC & $18(25.7)$ \\
\hline Others & $3(4.3)$ \\
\hline \multicolumn{2}{|l|}{ Lauren classification } \\
\hline Intestinal & $30(42.9)$ \\
\hline Diffuse & $40(57.1)$ \\
\hline \multicolumn{2}{|l|}{ Stage } \\
\hline IIA & $10(14.3)$ \\
\hline IIB & $10(14.3)$ \\
\hline IIIA & $4(5.7)$ \\
\hline IIIB & $8(11.4)$ \\
\hline IIIC & $18(25.7)$ \\
\hline IV & $20(28.6)$ \\
\hline \multicolumn{2}{|l|}{ Type of surgery } \\
\hline Total gastrectomy & $36(51.4)$ \\
\hline Subtotal gastrectomy & $34(48.6)$ \\
\hline \multicolumn{2}{|l|}{ Adjuvant therapy } \\
\hline Adjuvant chemotherapy & $66(94.3)$ \\
\hline Adjuvant radiotherapy & $2(2.9 \%)$ \\
\hline
\end{tabular}

High RhoJ expression is associated with poor survival outcome in GC patients

On Kaplan-Meier survival analysis, RhoJ-positive group displayed markedly poorer OS and RFS compared with RhoJ-negative group $(\mathrm{p}=0.001$ and 0.002 , respectively) (Figure $2 \mathrm{~A}$ and $2 \mathrm{~B}$ ).

Next, we further examined the prognostic value of high RhoJ expression with adjustments for other prognostic factors with a Cox's multivariate analysis (Table 3). Even after the adjustment, high RhoJ expression still conferred a significant impact on OS and RFS of GC patients $(p=0.038$ and $p=0.047$, respectively), indicating that it is an independent prognostic factor for OS and RFS. Collectively, these findings evidence that high RhoJ expression is a negative prognostic factor for the survival outcome of GC patients.

Table 2. Correlation between RhoJ expression and clinical parameters.

\begin{tabular}{|c|c|c|c|}
\hline & RhoJ-negative $(n=36)$ & RhoJ-positive $(n=34)$ & p-value \\
\hline Age & $61(33-76)$ & $59(33-83)$ & 0.865 \\
\hline Sex & & & 0.863 \\
\hline Male & $24(66.7)$ & $22(64.7)$ & \\
\hline Female & $12(33.3)$ & $12(35.3)$ & \\
\hline Histology & & & 0.043 \\
\hline WD and MD & $15(41.7)$ & $5(14.7)$ & \\
\hline PD and SRC & $19(52.8)$ & $28(82.4)$ & \\
\hline Others & $2(5.5)$ & $1(2.9)$ & \\
\hline Lauren classification & & & 0.007 \\
\hline Intestinal & $21(58.3)$ & $9(26.5)$ & \\
\hline Diffuse & $15(41.7)$ & $25(73.5)$ & \\
\hline Stage & & & 0.023 \\
\hline IIA & $7(19.4)$ & $3(8.8)$ & \\
\hline IIB & $8(22.2)$ & $2(5.9)$ & \\
\hline IIIA & $2(5.6)$ & $2(5.9)$ & \\
\hline IIIB & $5(13.9)$ & $3(8.8)$ & \\
\hline IIIC & $10(27.8)$ & $8(23.5)$ & \\
\hline IV & $4(11.1)$ & $16(47.1)$ & \\
\hline T stage & & & 0.411 \\
\hline $\mathrm{T} 2$ & 4 (11.1) & $4(11.8)$ & \\
\hline $\mathrm{T} 3$ & $10(27.8)$ & $4(11.8)$ & \\
\hline $\mathrm{T} 4 \mathrm{a}$ & $19(52.8)$ & $22(64.7)$ & \\
\hline $\mathrm{T} 4 \mathrm{~b}$ & $3(8.3)$ & $4(11.8)$ & \\
\hline N stage & & & 0.467 \\
\hline N0 & $8(22.2)$ & $3(8.8)$ & \\
\hline N1 & $9(25.0)$ & 7 (20.6) & \\
\hline $\mathrm{N} 2$ & $4(11.1)$ & $6(17.6)$ & \\
\hline $\mathrm{N} 3 \mathrm{a}$ & $13(36.1)$ & 14 (41.2) & \\
\hline $\mathrm{N} 3 \mathrm{~b}$ & $2(5.6)$ & $4(11.8)$ & \\
\hline M stage & & & 0.001 \\
\hline M0 & 32 (88.9) & $18(52.9)$ & \\
\hline M1 & 4 (11.1) & $16(47.1)$ & \\
\hline
\end{tabular}

Table 3. Prognostic factor for OS and RFS in multivariate Cox regression model.

\begin{tabular}{|c|c|c|c|c|}
\hline & OS & \multicolumn{3}{|c|}{ RFS } \\
\hline & HR $(95 \% \mathrm{CI})$ & p-value & HR $(95 \% \mathrm{CI})$ & p-value \\
\hline Age $(\geq 65$ vs. $<65)$ & $0.70(0.35-1.41)$ & 0.314 & $0.39(0.18-0.85)$ & 0.018 \\
\hline $\begin{array}{l}\text { RhoJ (Positive vs. } \\
\text { Negative) }\end{array}$ & $2.68(1.30-5.53)$ & 0.038 & $2.09(1.01-4.32)$ & 0.047 \\
\hline $\begin{array}{l}\text { Histology (PD, SRC vs. } \\
\text { WD, MD) }\end{array}$ & $1.44(0.72-2.88)$ & 0.302 & $1.56(0.78-3.12)$ & 0.213 \\
\hline Stage (III, IV vs. II) & $2.74(1.13-6.65)$ & 0.025 & $3.03(1.13-8.11)$ & 0.027 \\
\hline
\end{tabular}




\section{RhoJ overexpression enhanced GC cell migration and invasion in vitro}

To elucidate the role of RhoJ overexpression on the biologic behavior of GC cells, we established RhoJ-overexpressing (RhoJ-OV) cells by transfecting YCC-16 GC cell lines with RhoJ-expressing plasmid (Figure 3A). The overexpression of RhoJ in RhoJ-OV cells did not affect the proliferation of GC cells compared to empty-vector transfected control (RhoJ-WT) cells (Figure 3B). In addition, there was no difference in the proportion of $\mathrm{PH}^{+}$proliferating cells between RhoJ-OV and RhoJ-WT cells (Figures $3 \mathrm{C}$ and $3 \mathrm{D})$, indicating that RhoJ has negligible role in the regulation of GC cell proliferation. On the other hand, RhoJ-OV cells displayed $41.3 \%$ increase in cell motility across the membrane of cell culture inserts $(p=0.015)$ (Figures 3E and 3F). Moreover, RhoJ-OV cells had $60.4 \%$ enhanced invasion ability across the Matrigel $^{\mathrm{TM}}$ extracellular matrix compared to RhoJ-WT cells $(p=0.024)$ (Figures $3 G$ and $3 H)$. Taking these results together, we were able to confirm that RhoJ in GC cell plays an important role in the regulation of GC cell motility and invasiveness.

\section{Discussion}

Rho GTPases regulate diverse cellular processes such as cytoskeletal rearrangement, cell motility, cell-cycle progression, and transcription $[9,10]$. Though there are increasing number of preclinical evidences reporting the importance of Rho GTPases during tumor progression and metastasis [8-10], the clinical significance of Rho GTPases in human cancer is largely unknown, especially in GC. Only few studies reported increased expression of RhoA, RhoC, and Rac1 in GC and their prognostic role in GC $[10,18,19]$.

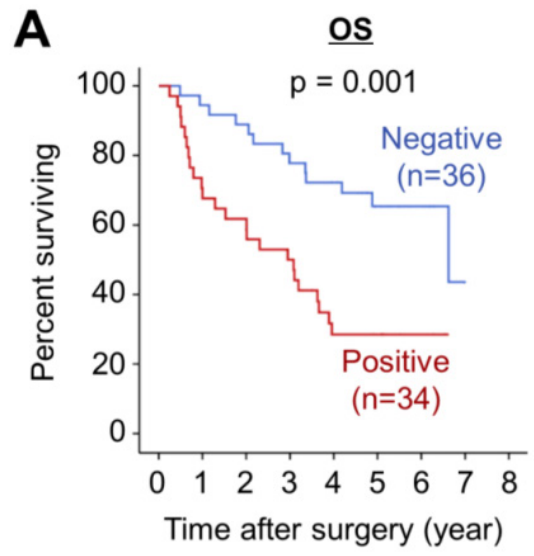

Inspired by these recent results in the role of RhoJ in melanoma [16,17], we explored whether RhoJ is also expressed in GC tissue. Intriguingly, in human GC, RhoJ was predominantly expressed in tumor cells with occasional expression in some part of tumor vasculatures. This finding implies that the cells responsible for RhoJ expression may vary according to the types of cancer. In GC, RhoJ expression in tumor cells seems to be more prevailing compared to endothelial RhoJ expression. Moreover, we examined whether RhoJ expression has a potential clinical value in GC patients. As a result, we were able to confirm the varied expression of RhoJ in GC cell lines and GC tissues, and could demonstrate that high RhoJ expression is a negative prognostic factor for the OS and RFS of GC patients after curative surgery. Even after adjustment for other prognostic factors, high RhoJ expression had significantly negative influence on survival outcome of GC patients. To our knowledge, this is the first study to demonstrate the independent prognostic value of RhoJ in human cancer.

We were also able to discover the possible mechanism in which RhoJ leads to the progression and metastasis of GC. Our in vitro experiments displayed the increased motility and invasiveness of GC cells where RhoJ was forcefully overexpressed by the transfection of RhoJ-expression vector compared to control GC cells, while there was no difference in terms of GC cell proliferation. This in vitro evidence suggests that the increased progression and metastasis observed in RhoJ-high GC patients might be attributed to the enhanced migratory and invasive ability of highly RhoJ-expressing GC cells.

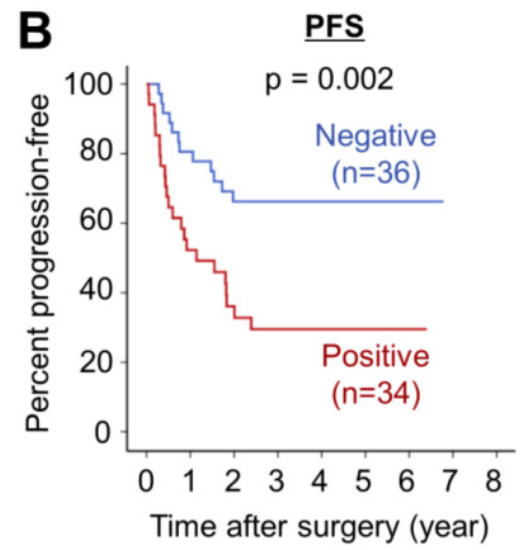

Figure 2. RhoJ expression is a negative prognostic factor for GC patients. (A) Kaplan-Meier analysis of overall survival (OS) in RhoJ-positive and RhoJ-negative GC patients. (B) Kaplan-Meier analysis of recurrence-free survival (RFS) in RhoJ-positive and RhoJ-negative GC patients. 


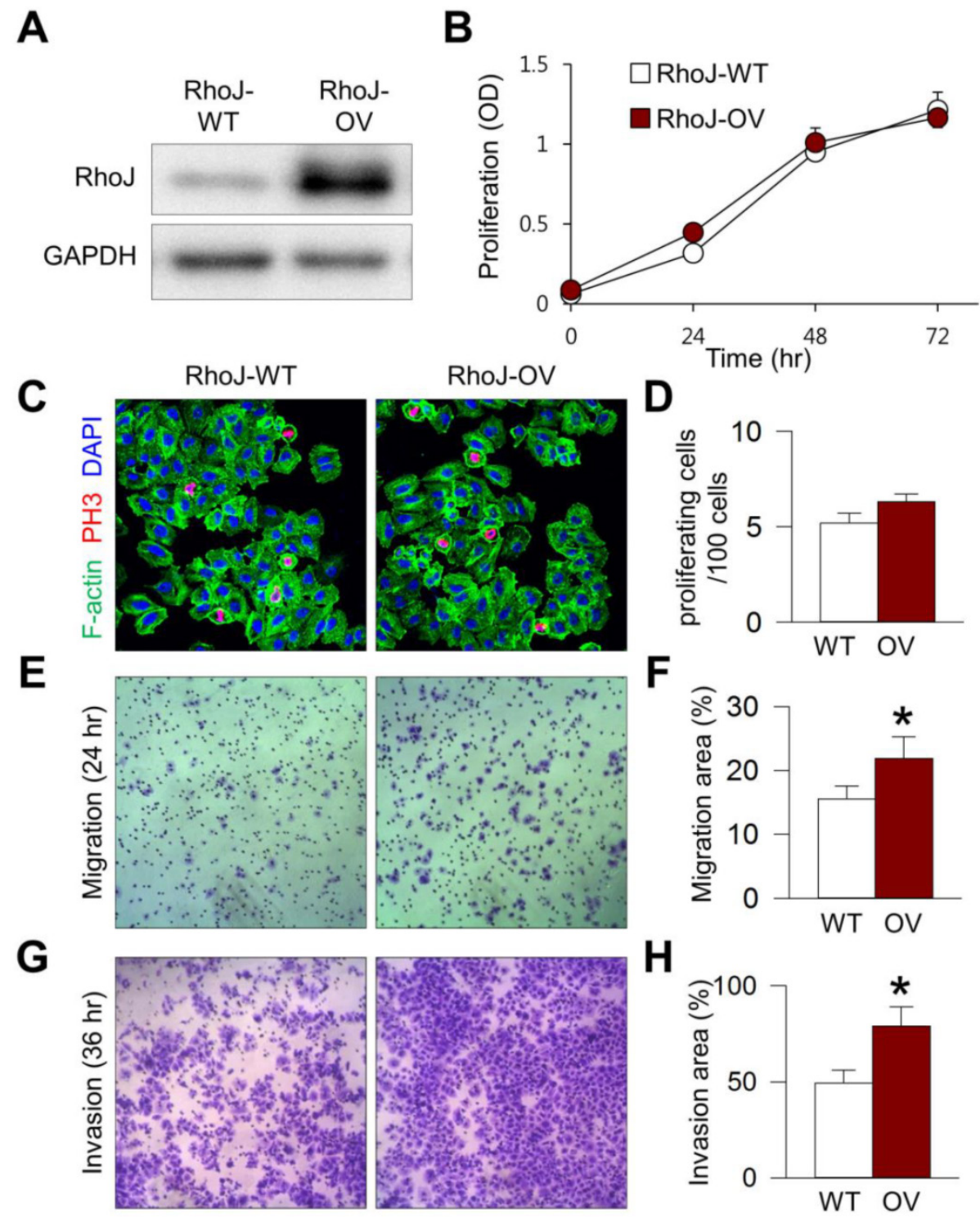

Figure 3. RhoJ-overexpressing GC cells have enhanced migratory and invasion ability. YCC-16 cells were transfected with either empty or RhoJ-expressing vectors using Lipofectamine. Unless otherwise denoted: Values are mean \pm standard deviations. $n=5$. * $<<0.05$ versus RhoJ-WT. (A) RhoJ expression level was compared by immunoblotting $24 \mathrm{hr}$ after transfection. RhoJ-overexpressing (RhoJ-OV) cells displayed markedly increased Rhol expression compared to control (RhoJ-WT) cells. (B) The effect of RhoJ expression on GC cell proliferation. Cell viability was evaluated using WST assay on the indicated time points after transfection. ( $C$ and $\mathrm{D}$ ) Images and quantification of $\mathrm{PH}^{+}$proliferating cells. ( $\mathrm{E}$ and $\mathrm{F}$ ) Images and quantification of $\mathrm{GC}$ cell migration across cell culture insert after $24 \mathrm{hr}$ of incubation. ( $\mathrm{G}$ and $\mathrm{H}$ ) Images and quantification of $\mathrm{GC}$ cell invasion across Matrigel extracellular matrix after $36 \mathrm{hr}$ of incubation.

Despite the novel findings of our study, one critical question that remained to be answered in the future is whether RhoJ is a feasible therapeutic target for the treatment of GC. For the moment, there is no direct evidence indicating the therapeutic value of RhoJ blockade in human cancer. However, previous studies employing animal tumor models could provide some hints to this question. The previous work that focused on endothelial RhoJ showed that the genetic deletion of host-derived RhoJ could suppress tumor progression and metastasis through the inhibition of tumor angiogenesis [15]. Besides, Ho et al. reported that the knockdown of RhoJ in melanoma cell with shRNA could delay melanoma growth and lymphatic metastasis [17]. Therefore, the strategy of targeting RhoJ has the potential of dual-targeting of tumor cells and tumor vessels simultaneously. This hypothesis has to be validated with animal models of GC when RhoJ inhibitors are developed and become available.

In conclusion, the current study presents evidence that RhoJ is positively associated with the regulation of tumor cell motility and invasiveness in GC. Moreover, we were able to demonstrate that high RhoJ expression in GC is an independent negative prognostic factor after gastrectomy. Further studies are required to validate the therapeutic role of RhoJ in GC by using RhoJ-specific inhibitor. 


\section{Acknowledgement}

This research was supported by a grant of the Research Driven Hospital R\&D project, funded by the CHA Bundang Medical Center (grant number: BDCHA R\&D 2015-37). This research was supported by a grant of the Korea Health Technology R\&D Project through the Korea Health Industry Development Institute (KHIDI), funded by the Ministry of Health \& Welfare, Republic of Korea (HI13C2096).

\section{Conflicts of Interest}

All authors disclosed no potential conflicts of interest.

\section{References}

1. Jemal A, Bray F, Center MM, Ferlay J, Ward E, Forman D. Global cancer statistics. CA: a cancer journal for clinicians. 2011; 61: 69-90.

2. Jung K-W, Won Y-J, Kong H-J, Oh C-M, Shin A, Lee J-S. Survival of Korean Adult Cancer Patients by Stage at Diagnosis, 2006-2010: National Cancer Registry Study. Cancer Research and Treatment. 2013; 45: 162-71.

3. Shah MA, Kelsen DP. Gastric cancer: a primer on the epidemiology and biology of the disease and an overview of the medical management of advanced disease. Journal of the National Comprehensive Cancer Network. 2010; 8: 437-47.

4. Cho JY. Molecular Diagnosis for Personalized Target Therapy in Gastric Cancer. Journal of gastric cancer. 2013; 13: 129-35.

5. Hanahan D, Weinberg RA. Hallmarks of cancer: the next generation. Cell. 2011; 144: 646-74.

6. Friedl P, Alexander S. Cancer invasion and the microenvironment: plasticity and reciprocity. Cell. 2011; 147: 992-1009.

7. Sahai E, Marshall CJ. RHO-GTPases and cancer. Nature Reviews Cancer. 2002; 2: 133-42.

8. Heasman SJ, Ridley AJ. Mammalian Rho GTPases: new insights into their functions from in vivo studies. Nature reviews Molecular cell biology. 2008; 9 : 690-701.

9. Mardilovich K, Olson MF, Baugh M. Targeting Rho GTPase signaling for cancer therapy. Future oncology. 2012; 8: 165-77.

10. Vega FM, Ridley AJ. Rho GTPases in cancer cell biology. Febs Letters. 2008; 582: 2093-101.

11. Leszczynska K, Kaur S, Wilson E, Bicknell R, Heath VL. The role of RhoJ in endothelial cell biology and angiogenesis. Biochemical Society Transactions. 2011; 39: 1606-11.

12. Vignal E, De Toledo M, Comunale F, Ladopoulou A, Gauthier-Rouvière C, Blangy A, et al. Characterization of TCL, a new GTPase of the Rho family related to TC10 and Cdc42. Journal of Biological Chemistry. 2000; 275: 36457-64.

13. Kaur S, Leszczynska K, Abraham S, Scarcia M, Hiltbrunner S, Marshall CJ, et al. RhoJ/TCL regulates endothelial motility and tube formation and modulates actomyosin contractility and focal adhesion numbers. Arteriosclerosis, thrombosis, and vascular biology. 2011; 31: 657-64.

14. Yuan L, Sacharidou A, Stratman AN, Le Bras A, Zwiers PJ, Spokes K, et al. RhoJ is an endothelial cell-restricted Rho GTPase that mediates vascular morphogenesis and is regulated by the transcription factor ERG. Blood. 2011; 118: $1145-53$.

15. Kim C, Yang H, Fukushima Y, Saw PE, Lee J, Park J-S, et al. Vascular RhoJ Is an Effective and Selective Target for Tumor Angiogenesis and Vascular Disruption. Cancer cell. 2014; 25: 102-17.

16. Ho H, Aruri J, Kapadia R, Mehr H, White MA, Ganesan AK. RhoJ regulates melanoma chemoresistance by suppressing pathways that sense DNA damage. Cancer research. 2012; 72: 5516-28.

17. Ho H, Soto Hopkin A, Kapadia R, Vasudeva P, Schilling J, Ganesan AK. RhoJ modulates melanoma invasion by altering actin cytoskeletal dynamics. Pigment cell \& melanoma research. 2013; 26: 218-25.

18. Pan Y, Bi F, Liu N, Xue Y, Yao X, Zheng Y, et al. Expression of seven main Rho family members in gastric carcinoma. Biochemical and biophysical research communications. 2004; 315: 686-91.

19. Kondo T, Sentani K, Oue N, Yoshida K, Nakayama H, Yasui W. Expression of RHOC is associated with metastasis of gastric carcinomas. Pathobiology. 2003; 71: $19-25$. 